\title{
SUPPLEMENTARY MATERIALS: PARALLEL PARTIAL GAUSSIAN PROCESS EMULATION FOR COMPUTER MODELS WITH MASSIVE OUTPUT
}

\author{
By Mengyang Gu and James O. Berger
}

1. Periodic Folding. The input $\phi$ in TITAN2D is periodic with values ranging from 0 to $2 \pi$. Spiller et al. (2014) introduces a formal way to deal with such an input, using a circular correlation function with the "periodic folding" form

$$
c^{c i r}\left(\phi_{1}, \phi_{2}\right)=\sum_{k=-\infty}^{\infty} c\left(\phi_{1}-\phi_{2}+2 k \pi\right) .
$$

Based on Bochner's theorem, it is easy to show that $c^{c i r}(, .$,$) is a valid 2 \pi$ periodic correlation function with spectral representation,

$$
c^{c i r}\left(\phi_{1}, \phi_{2}\right)=\frac{c_{0}}{2 \pi}+\frac{1}{\pi} \sum_{n=1}^{\infty} c_{n} \cos \left(n\left(\phi_{1}-\phi_{2}\right)\right) .
$$

By using the power exponential correlation $c\left(\phi_{1}-\phi_{2}\right)=\exp \left(-\left(\frac{\left|\phi_{1}-\phi_{2}\right|}{\gamma_{\phi}}\right)^{\alpha_{\phi}}\right)$, it is shown in Spiller et al. (2014) that the coefficient $c_{n}$ is

$$
c_{n}=\left\{\begin{array}{ll}
2 \gamma_{\phi} /\left(\gamma_{\phi}^{2}+n^{2}\right) & \alpha_{\phi}=1 \\
e^{-n^{2} / 4 \gamma_{\phi}} \sqrt{\pi / \gamma_{\phi}} & \alpha_{\phi}=2 \\
2 \int_{0}^{\infty} e^{-\gamma_{\phi} x^{\alpha}} \cos (n x) d x & 1<\alpha_{\phi}<2
\end{array} .\right.
$$

We choose $c^{\operatorname{cir}}(\cdot, \cdot)$ to be the ten term approximation to (1.2), with $\alpha_{\phi}=$ 2 so that the $c_{n}$ are available in closed form. This has the advantage of guaranteeing a positive definite covariance function [Spiller et al. (2014)], whereas approximating (1.1) would not guarantee positive definiteness.

2. close to interpolation by PP GaSP with a nugget. One of the advantages of the original $\mathrm{PP}$ emulator is that it is an interpolator of the simulator at the input design points; this will no longer be true of the 
PP emulator with a nugget. It is thus useful to examine extent of lack of interpolation of the latter, using the following measure:

$$
Q^{\mathscr{D}}=1-\frac{\sum_{j=1}^{k} \sum_{i=1}^{n}\left(y_{j}\left(\mathbf{x}_{i}^{\mathscr{D}}\right)-\hat{y}_{j}\left(\mathbf{x}_{i}^{\mathscr{D}}\right)\right)^{2}}{\sum_{j=1}^{k} \sum_{i=1}^{n}\left(y_{j}\left(\mathbf{x}_{i}^{\mathscr{D}}\right)-\overline{\mathbf{y}}^{\mathscr{D}}\right)^{2}},
$$

where $\hat{y}_{j}\left(\mathbf{x}_{i}^{\mathscr{D}}\right)$ is the posterior mean of the PP GaSP emulator with nugget at design input $\mathbf{x}_{i}^{\mathscr{D}}, y_{j}\left(\mathbf{x}_{i}^{\mathscr{D}}\right)$ is the exact simulator value and the summation is over all design input values and all output coordinates. The measure is scaled by the sum of square deviations of the simulator outputs from the overall output mean $\overline{\mathbf{y}}^{\mathscr{D}}$, so that the measure is unitless; the value of 1 would indicate perfect interpolation.

For the PP-emulator with nugget that was implemented above based on $n=50$ design simulator runs, the value of the measure, over the 50 runs and $k=23,040$ output coordinates, is $Q^{\mathscr{D}} \approx 0.998$. Being very close to 1 , this indicates that the PP-emulator with nugget is almost an interpolator.

3. Smoothing the draws of the PP GaSP emulator. For some uncertainty quantification tasks, actual draws from the PP GaSP are required, e.g., draws of $\left(y_{1}\left(\mathbf{x}^{*}\right), \ldots, y_{k}\left(\mathbf{x}^{*}\right)\right)$. Because of the independence assumption in the PP emulator, nearby coordinates can have quite different values in these draws, which might have a detrimental effect in the uncertainty quantification task. In this section, we introduce an emulator that has a spatial covariance structure $\boldsymbol{\Sigma}$, which allows for smoothing over nearby coordinates and yet has the exact posterior mean and variance as the PP GaSP Emulator. (That it has the same mean is automatic, as shown in Theorem 1; thus it is only required for the new emulator to yield the same $\hat{\sigma}_{j}^{2}$ as the PP emulator.)

The idea is relatively simple: we divide the coordinate space into blocks of small size $s<n-q$ ( $s=4$ and $s=16$ will be used in the illustrations). The correlation between different blocks will be set to zero while, correlation is allowed within each block. The mean for each block is an $n \times s$ matrix $\boldsymbol{\theta}_{b}$ and the block covariance matrix, $\boldsymbol{\Sigma}_{b}$, is $s \times s$.

For each block $b, 1 \leq b \leq B$, we will mimic the analysis of Conti and OHagan (2010), assigning the objective prior

$$
\pi\left(\boldsymbol{\theta}_{b}, \boldsymbol{\Sigma}_{b} \mid \boldsymbol{\gamma}, \nu\right) \propto\left|\boldsymbol{\Sigma}_{b}\right|^{-(s+1) / 2}
$$

and marginalizing out $\boldsymbol{\Sigma}_{b}$. Then, for each block, the predictive distribution 
is

$$
\mathbf{y}_{b}\left(\mathbf{x}^{*}\right) \mid \mathbf{y}_{b}^{\mathscr{D}}, \hat{\gamma} \sim t\left(\hat{y}_{b}\left(\mathbf{x}^{*}\right), c^{* *} \hat{\boldsymbol{\Sigma}}_{b}, n-q\right)
$$

where $\mathbf{y}_{b}^{\mathscr{D}}$ is the $n \times s$ matrix of outputs at the observed inputs and

$$
\begin{aligned}
\hat{y}_{b}\left(\mathbf{x}^{*}\right) & =\mathbf{h}\left(\mathbf{x}^{*}\right) \hat{\boldsymbol{\theta}}_{b}+\mathbf{r}^{T}\left(\mathbf{x}^{*}\right) \mathbf{R}^{-1}\left(\mathbf{y}_{b}^{\mathscr{D}}-\mathbf{h}\left(\mathbf{x}^{\mathscr{D}}\right) \hat{\boldsymbol{\theta}}_{b}\right) \\
\hat{\boldsymbol{\Sigma}}_{b} & =\frac{\left(\mathbf{y}_{b}^{\mathscr{D}}-\mathbf{h}\left(\mathbf{x}^{\mathscr{D}}\right) \hat{\boldsymbol{\theta}}_{b}\right)^{T} \mathbf{R}^{-1}\left(\mathbf{y}_{b}^{\mathscr{D}}-\mathbf{h}\left(\mathbf{x}^{\mathscr{D}}\right) \hat{\boldsymbol{\theta}}_{b}\right)}{n-q}
\end{aligned}
$$

with $\hat{\boldsymbol{\theta}}_{b}=\left(\mathbf{h}^{T}\left(\mathbf{x}^{\mathscr{D}}\right) \boldsymbol{R}^{-1} \mathbf{h}\left(\mathbf{x}^{\mathscr{D}}\right)\right)^{-1} \mathbf{h}^{T}\left(\mathbf{x}^{\mathscr{D}}\right) \boldsymbol{R}^{-1} \mathbf{y}_{b}^{\mathscr{D}}$. Note that the posterior mean and variance are indeed the same as those from the PP emulator at each coordinate, but now there will be smoothing within each block. This sampling algorithm is summarized as Algorithm 1.

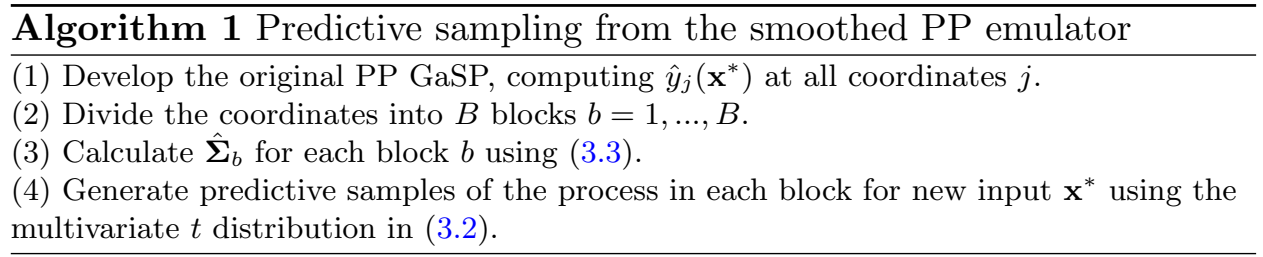

The only additional computational cost here is computation of the block covariance matrix $\hat{\boldsymbol{\Sigma}}_{b}$ and generating draws from the multivariate student $t$ distribution, neither of which is very expensive unless $s$ becomes large. But this is being done $B$ times, which will be huge since $k$ is huge; thus we use only moderate values of $s$, such as 4 and 16 .

There is one seemingly incoherent aspect of Algorithm 1, namely the use of the original PP GaSP - not the spatially augmented GaSP - to estimate the correlation parameters $\gamma$. This is intuitively reasonable, because $\gamma$ arises only in the input correlation part of the process, i.e., the original PP GaSP. Indeed, when we tried to use the likelihood from the spatially augmented GaSP to estimate $\gamma$, the results were considerably worse, presumably because the new spatial parameters allowed for additional confounding to arise, without providing sharper inferences about $\gamma$. Finally, note that we also considered a variety of alternate structures and priors for $\boldsymbol{\Sigma}$, including an autoregressive structure [Farah et al. (2014)], a sparse graphical model through a conditional way [Dobra et al. (2004)] and jointly through the hyper inverse Wishart prior [Carvalho et al. (2007); Wang and West (2009)], none of which improves emulation results of the PP GaSP (evaluated by the criteria discussed in Section 5). 


\begin{tabular}{rrrrr}
\hline & PP sample & $2 \times 2$ Block sample & $4 \times 4$ Block sample & PP emulator mean \\
\hline$\Delta$ & $35.6 \%$ & $26.8 \%$ & $19.5 \%$ & $0.96 \%$ \\
\hline
\end{tabular}

TABLE 1

Percent under smoothing of samples from various emulators for Montserrat Island, for $n^{*}=633$ held-out testing points and 23,040 grid points (coordinates).

To evaluate the local spatial smoothness of the spatially augmented GaSP, we use the measure

$$
\Delta=\left(\frac{\sum_{t=1}^{n^{*}} \sum_{i \in n e(j), 1 \leq i, j \leq k}\left|\tilde{y}_{i}\left(\mathbf{x}_{t}^{*}\right)-\tilde{y}_{j}\left(\mathbf{x}_{t}^{*}\right)\right|}{\sum_{t=1}^{n^{*}} \sum_{i \in n e(j), 1 \leq i, j \leq k}\left|y_{i}\left(\mathbf{x}_{t}^{*}\right)-y_{j}\left(\mathbf{x}_{t}^{*}\right)\right|}-1\right),
$$

where $\tilde{y}_{i}\left(\mathbf{x}_{t}^{*}\right)$ is the sample output at coordinate $i$ from the new GaSP at held-out input $\mathbf{x}_{t}^{*}$, and the outer sums are over the $n^{*}$ held-out points; and the inner sums are over pairs of neighboring coordinate points $i$ and $j$, the neighborhood set being defined as the the coordinates that are in the same predefined square block with the size of $s$. The results of $2 \times 2$ and $4 \times 4$ block have been shown in Table 1 . This measures the average smoothness, with respect to neighbors, of the sampled GaSP compared with the actual simulator. If $\Delta$ is positive, it means there is not enough smoothing while a negative $\Delta$ means over smoothing; and its numerical value can be interpreted as the percent of under or over smoothing.

First, note that the PP emulator mean is within $1 \%$ of the smoothness of the actual simulator, supporting our earlier statement that the PP emulator would inherit the smoothness of the simulator. In contrast, draws from the PP emulator are $35.6 \%$ more variable than the simulator, a clear lack of appropriate smoothness. Using the block spatially augmented emulators did improve the smoothness with the $4 \times 4$ block emulator essentially cutting the under smoothing by half. Clearly much bigger blocks would be needed to drive the smoothness of emulator draws to be closer to the smoothness of the simulator, but that would significantly impact the computational cost.

\section{References.}

Carvalho, C. M., West, M. et al. (2007). Dynamic matrix-variate graphical models. Bayesian analysis 2 69-97.

Conti, S. and OHAGAn, A. (2010). Bayesian emulation of complex multi-output and dynamic computer models. Journal of statistical planning and inference 140 640-651.

Dobra, A., Hans, C., Jones, B., Nevins, J. R., Yao, G. and West, M. (2004). Sparse graphical models for exploring gene expression data. Journal of Multivariate Analysis $90196-212$.

Farah, M., Birrell, P., Conti, S. and Angelis, D. D. (2014). Bayesian Emulation and Calibration of a Dynamic Epidemic Model for A/H1N1 Influenza. Journal of the American Statistical Association 109 1398-1411. 
Spiller, E. T., Bayarri, M., Berger, J. O., Calder, E. S., Patra, A. K., PitMAN, E. B. and Wolpert, R. L. (2014). Automating Emulator Construction for Geophysical Hazard Maps.

WANG, H. and WEST, M. (2009). Bayesian analysis of matrix normal graphical models. Biometrika 96 821-834. 